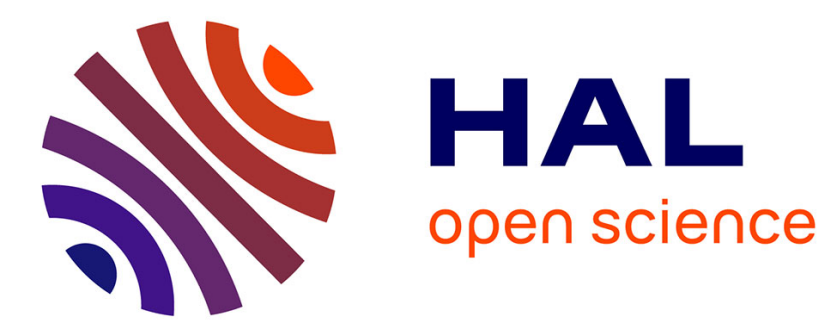

\title{
Évolutions réglementaires et investissements directs étrangers au Japon
}

Philippe Barbet, Nathalie Coutinet

\section{To cite this version:}

Philippe Barbet, Nathalie Coutinet. Évolutions réglementaires et investissements directs étrangers au Japon. Revue Internationale de Droit Economique, 2001, 4ème trimestre, pp.22. halshs-00206130

\section{HAL Id: halshs-00206130 \\ https://shs.hal.science/halshs-00206130}

Submitted on 16 Jan 2008

HAL is a multi-disciplinary open access archive for the deposit and dissemination of scientific research documents, whether they are published or not. The documents may come from teaching and research institutions in France or abroad, or from public or private research centers.
L'archive ouverte pluridisciplinaire HAL, est destinée au dépôt et à la diffusion de documents scientifiques de niveau recherche, publiés ou non, émanant des établissements d'enseignement et de recherche français ou étrangers, des laboratoires publics ou privés. 


\title{
Évolutions réglementaires et investissements directs étrangers au Japon
}

\author{
Philippe BARBET ${ }^{1}$ \\ Nathalie COUTINET ${ }^{2}$
}

\section{Résumé}

Cette contribution présente une évaluation du processus d'ouverture du marché japonais aux investissements directs étrangers (IDE) et en particulier aux opérations de fusions et d'acquisitions (F\&A). Après une analyse de ces réformes distinguées selon leur influence sur les investissements étrangers nous étudions, afin d'apprécier leur impact réel sur les investissements étrangers, les flux d'IDE entrants et le mouvement de fusions et d'acquisitions au Japon.

Dans un premier temps, nous nous attachons à dresser un bilan des réformes réglementaires engagées depuis le milieu des années 80 en distinguant les reformes d'ordre général, ayant une influence indirecte sur les IDE, des réformes ciblées plus spécifiquement sur l'ouverture aux capitaux étrangers. Ensuite, nous évaluons les résultats de ce processus d'ouverture à partir d'un examen précis des flux d'investissements directs entrants et des opérations de fusions et d'acquisitions. Une analyse sectorielle de ces flux et de ces opérations permet, enfin, de confirmer que les secteurs ayant connu, en plus des réformes générales, des réformes spécifiques sont ceux qui reçoivent le plus d'investissements étrangers.

\section{Abstract}

In this paper, we study the access of Japanese domestic market by Foreign Direct Investment (FDI) and specially Merger and Acquisitions (M\&A). We first analyse the legal reforms in Japan during the last fifteen years and their direct and indirect impact on "out- in" FDI flows and M\&A. In the first part of the paper, we examine the major changes in the legal framework in Japan and their direct and indirect consequences on FDI and M\&A. Then we evaluate the consequences of this new legal framework on FDI flows and M\&A in Japan both at the geographic and industry level. We show that when specific legal measures and deregulation processes affect specific industries, the flow of FDI and M\&A are more important than in other industries.

\footnotetext{
${ }^{1}$ Université Paris-Nord et CEPN - CNRS Faculté de Droit et de Sciences Politiques Université de Paris 13. 99 avenue J.B.Clément 93430 Villetaneuse - France. barbet@seg.univ-paris13.fr

${ }^{2}$ GEAPE Université d'Angers et CEPN - CNRS Faculté d'économie, de droit et de gestion Université d'Angers 13 allée François Mitterrand BP 363349036 Angers Cedex 01-France. nathalie.coutinet@univ-angers.fr
} 


\section{Introduction}

La question de l'ouverture du marché japonais aux importations et aux capitaux étrangers est particulièrement sensible depuis la fin des années 70, période qui consacre le caractère structurel de l’excédent commercial japonais [Lawrence 1987, Saxonhouse 1993]. Les autres pays développés, et particulièrement les Etats-Unis, ont engagé dans les années 80 de difficiles négociations ${ }^{3}$, souvent accompagnées de menaces rétorsions commerciales ${ }^{4}$, pour contraindre les pouvoirs publics japonais à modifier certaines modalités de régulation de leur économie accusées de faire office de barrières à l’entrée sur le marché japonais. Le processus d’ouverture aux échanges que connaît l'économie japonaise depuis le début des années 90 résulte donc à la fois de l'adoption volontaire des normes de fonctionnement de l'économie qui se diffusent dans les pays industrialisés mais aussi de pressions commerciales de plus en plus fortes. Ainsi, les réformes de structures ont été au centre des négociations américanojaponaises dites SII (Structural Impediment Initiatives talks) qui demandaient en particulier la mise en place d'une réforme foncière au Japon pour faire baisser les prix de l'immobilier et une modification profonde des réseaux de distribution. Le gouvernement japonais a également accepté, en 1991, d’ouvrir à la concurrence des activités réglementées et protégées comme les télécommunications, la distribution d’énergie et les services financiers. En 1993, le « JapanUS framework for a New Economic Partnership » a élargi le spectre de la déréglementation sectorielle à huit activités liées au commerce et à l'investissement international. Avec les Européens, les discussions bilatérales ont conduit à un accord en 1995 qui se limitait à des mesures de déréglementation et d'ouverture dans la construction automobile et les pièces détachées.

A la suite de ce processus d'ouverture «négocié » avec ses principaux partenaires, la crise et l'arrêt de la croissance au Japon ont incontestablement modifié l'approche japonaise des relations commerciales internationales. En 1998, le MITI (Ministry of International Trade and Industry) reconnaît officiellement le rôle moteur non seulement des exportations mais aussi des importations et de l'entrée des firmes étrangères pour faciliter la reprise de la croissance au Japon (White Paper on Trade and Industry). L’ouverture du marché japonais aux

\footnotetext{
${ }^{3}$ En 1986 les négociations MOSS (Markets Orientated Sectors Specific) tentaient de définir des modalités d'ouverture de certains secteurs. En 1987, la commission « Makewa » préconisait de faciliter l'accès du marché japonais aux firmes étrangères.

${ }^{4}$ En 1989 le Japon est soumis à l'article 301 du Trade Act américain et il lui est demandé d'ouvrir son marché aux firmes étrangères
} 
importations et en particulier aux investissements étrangers a été facilitée par le changement d'approche que nous avons résumé ci-dessus mais un certain nombre de mesures, plus ponctuelles et plus ciblées, ont sans doute joué un rôle majeur dans ce processus.

Cet article présentera une évaluation de ce processus d'ouverture en se centrant sur le cas des investissements directs des entreprises étrangères sur le marché japonais. Dans un premier temps, nous analyserons la dynamique réglementaire en cours au Japon en distinguant entre les différents types de mesures facilitant l'ouverture du marché japonais. Dans un second temps, nous présenterons une évaluation de ces mesures en terme de flux d'investissements directs entrants et en particulier de fusions \& acquisitions.

\section{Les principales évolutions réglementaires facilitant l'ouverture aux investissements étrangers}

Au début des années 90, le Japon a engagé une série de réformes visant à améliorer l'accès du marché japonais aux firmes étrangères [OCDE 1999]. Les différents types de mesures qui ont été adoptées répondent aux multiples formes de fermeture du marché. Ainsi, certaines, comme le "deregulation action program" de 1995-1998 (devenu le "japanese deregulation program" pour la période 1998-2001), sont d'ordre général alors que d'autres ont pour objectif d'améliorer la transparence des procédures d'accès au marché ${ }^{5}$ ou de simplifier et d'harmoniser au niveau international les processus de certification et de standardisation ${ }^{6}$. Enfin, certaines portent spécifiquement sur certains types d'opérations (les IDE par exemple) ou sur des secteurs particuliers (la finance ou les télécommunications). Il convient enfin de noter que les différentes mesures adoptées ont souvent été précédées de l'élaboration de rapport et de documents ${ }^{7}$ ou de la création d'institutions ayant pour objectif soit d'inciter aux réformes soit d'informer les firmes étrangères ${ }^{8}$.

Nous avons cherché à ordonner l'ensemble des mesures prises en les regroupant dans un tableau (voir tableau 1 page suivante) et en distinguant d'une part les rapports ou institutions

\footnotetext{
${ }^{5}$ The administrative procedure law de 1998

${ }^{6}$ The policy actions on market issues adoptée par l'OMA en 1997

${ }^{7}$ Par exemple le "statement on mergers and acquisitions" du JIC de 1996 précède les mesures prises en faveur des fusions et acquisitions.

${ }^{8}$ La création du FIND Foreign Investment in Japan Development Corporation) s'inscrit dans la loi de 1992.
} 
et les mesures prises et d'autre part les mesures portant directement sur les IDE entrants de celles plus générales qui contribuent à les faciliter. 


\section{Tableau $\mathbf{n}^{\circ} 1$ : Rapports d'orientation, mesures directes et indirectes concernant l'ouverture aux investissements étrangers}

\begin{tabular}{|c|c|c|}
\hline & Influence directe sur les IDE & Influence indirecte sur les IDE \\
\hline $\begin{array}{l}\text { Rapports } \\
\text { Documents } \\
\text { d'orientation } \\
\text { Institutions }\end{array}$ & $\begin{array}{l}1982 \text { : OTO } \\
1993 \text { : Création du FIND (Foreign Investment in Japan Development } \\
\text { Corporation) } \\
\quad 1997 \text { Rapport du FIND sur les F\&A } \\
1994 \text { : Création Japan Investment Council (JIC) } \\
\quad 1995 \text { mission d'encouragement des IDE } \\
\quad 1996 \text { Statement on M\&A } \\
\quad 1997 \text { Rapport sur les IDE entrants } \\
\quad 1999 \text { Rapport proposant } 7 \text { recommandations pour } \\
\text { promouvoir les FDI au Japon } \\
\text { A partir de } 1996 \text {; le JETRO, le FIND et la japan development bank } \\
\text { fournissent de l'information sur les investissements entrants par des } \\
\text { séminaires etc. }\end{array}$ & $\begin{array}{l}1958 \text { : Création du JETRO } \\
1997 \text { : Action plan for economic structural reform (200 propositions pour améliorer l'accès au } \\
\text { marché) } \\
1998 \text { : MITI (Ministry of International Trade and Industry) White paper on trade and industry } \\
1998: \text { Three years programme for the promotion of regulation. Révisé en } 1999 \text { sur les relations } \\
\text { fournisseurs/distributeurs. }\end{array}$ \\
\hline Mesures & $\begin{array}{l}1992 \text { Révision de la «Foreign exchange law » pour faciliter les IDE } \\
\text { entrants } \\
1992 \text { : Import and inward investment law, étendue en } 1996 \text { jusqu'en } \\
2006 \text {. } \\
\text { En } 1995 \text { elle devient : Special law on extraordinary measures for } \\
\text { promoting imports and facilitation of inward investistment } \\
\quad 1992 \text { création des Foreign Acces Zones. Modifiés en } 1995 \text { et } \\
\text { étendues pour } 10 \text { ans. } \\
1996 \text { : The Industrial structure improvement fund garantit les prêts } \\
\text { certifiés par la loi Import and inward investment } \\
\text { 1996: Le MITI baisse les taux d'intérêt demandés par la Japan } \\
\text { development bank pour les investissements étrangers dans les hautes } \\
\text { technologies. } \\
\text { 1996: Programme de prêts pour faciliter les alliances et pour } \\
\text { promouvoir les F\&A. En } 1997 \text { la loi est étendue pour des firmes ayant } \\
\text { au moins 33\% de capital étranger (au lieu de 50\%) } \\
\text { 1998 Mesures de promotion des investissements étrangers } \\
\text { 2001 : Nouveau système d'imposition sur les scissions, fusions et } \\
\text { investissements liés aux restructurations d'entreprises }\end{array}$ & $\begin{array}{l}1992 \text { : Remplacement de la notification préalable par un contrôle ex post des opérations } \\
\text { d'investissement } \\
\text { Depuis } 1994 \text { : Diverses mesures pour ouvrir les marchés publics aux firmes étrangères } \\
1995-98 \text { : Deregulation action program qui devient le japanese deregulation program 98-2001 } \\
1995 \text { : Le «japanese patent office " prend des mesures dont la compatibilité internationale des } \\
\text { réglementations, la réduction du temps d'examen du dossier de brevets à } 24 \text { mois puis à } 12 \text { en } \\
2000 \\
1996 \text { : Insurance Agreement qui déréglemente le secteur des assurances } \\
1997 \text { : Office Market Access (OMA) adopte le "policy actions on market access issues as } \\
\text { concerns standards certification and others" } \\
1997 \text { Social and economic action plan (reconnaissance mutuel de normes) } \\
1997 \text { : Modification du code commercial pour simplification des procédures de fusion } \\
1997 \text { : Program for changing and creating a new economic structure qui comprend un } \\
\text { programme de dérégulation dans certains secteurs (énergie, télécom, finance etc.) } \\
1998 \text { : Loi sur les grandes surfaces (qui se substitue à la loi de 1990). Rentre en vigueur en juin } \\
2000 \\
1998 \text { Amendement de la loi anti monopole concernant les F\&A (effective le 01-1999) } \\
1998 \text { Administrative procedure law (Transparence des procédures) } \\
1999 \text { : Modification du code commercial concernant les procédures de cession d'actifs }\end{array}$ \\
\hline
\end{tabular}




\subsection{Les mesures influençant indirectement les IDE.}

Les deux principales mesures prises par les autorités japonaises et antérieures au programme de déréglementation de l'économie de 1995 sont d'une part la création du JETRO en 1958 qui offre des services consultatifs et qui compile et fournit de l'information liée aux IDE entrants et d'autre part la publication annuelle par le MITI de rapports dans lesquels l'attention portée aux IDE est de plus en plus importante.

Le programme de déréglementation de 1995-1998 suivit de celui de 1998-2001 est de grande ampleur et porte sur de nombreux aspects de l'économie japonaise. Il consiste en un ensemble de mesures visant à simplifier et à réduire les procédures administratives pesant sur les firmes étrangères et japonaises. Un aspect important de ce programme est l'ouverture aux IDE qui doit permettre aux offreurs étrangers d'entrer sur le marché national sans subir de discrimination ou de coûts d’accès anormalement élevés.

Parmi les mesures adoptées, certaines concernent la compatibilité internationale des réglementations sur les brevets (1995), la reconnaissance internationale des normes et standards (social and economic action plan de 1997) ou l'amélioration des conditions d'accès au marché (action plan for economic structural reform de 1997).

La loi sur les grandes surfaces (élaborée en 1998 et entrée en application en juin 2000) illustre bien l'importance de ces mesures. Ce sont dorénavant les municipalités et non plus l'État qui décident de l'opportunité de l'implantation d'une grande surface en fonction de règles liées au respect de l'environnement du magasin (bruit, embouteillages et gestion des déchets). La réglementation précédente protégeait les petites surfaces en empêchant de fait toute nouvelle ouverture sur un territoire ${ }^{9}$. La nouvelle réglementation permettra aux firmes étrangères de pénétrer plus facilement le marché japonais de la distribution.

\footnotetext{
${ }^{9}$ Cette loi devrait engendrer des modifications importantes du système de distribution au Japon. Les relations privilégiées entre fournisseurs et distributeurs (issues des Keiretsu) devraient laisser la place à des pratiques concurrentielles plus proches de celles constatées aux Etats-Unis et en Europe. Des firmes étrangères ont déjà pénétré avec succès le marché japonais (Toys' R Us et GAP pour les Etats-Unis, Carrefour pour la France).
} 
Concernant plus précisément les fusions et acquisitions plusieurs modifications réglementaires sont intervenues ${ }^{10}$. D'abord, en 1997, un amendement au code commercial est adopté dans le but de simplifier les procédures concernant les fusions. Puis, en 1998, un amendement de la loi anti-monopole introduit un seuil de déclaration des F\&A (et supprime la déclaration systématique). Les fusions impliquant des firmes étrangères doivent être déclarées si les ventes au Japon des filiales de compagnies étrangères dépassent 1 milliard de yens (pour les firmes japonaises le seuil est de 10 milliards de yens). De plus les fusions entre une firme et un sous-traitant n’ont plus à être déclarées. Enfin, la loi de 1999, concernant le code commercial, a modifié de façon substantielle le droit des sociétés. Les actionnaires minoritaires d'une société ne peuvent plus s'opposer aux cessions d'actifs. Cette loi permet aux actionnaires de mieux contrôler la gestion de la filiale et de la maison-mère. Plus récemment, la réforme fiscale de 2001, a complété cette loi en adoptant un nouveau système d'imposition concernant les scissions, fusions et investissements liés aux restructurations d'entreprises.

Les lois les plus récentes prises par le gouvernement japonais sont des lois de déréglementation sectorielle et d'encouragement des investissements dans les nouvelles technologies de l'information et de la communication.

La politique de réformes entreprise par le gouvernement japonais est axée autour de trois actions : la déréglementation (notamment sectorielle), la politique de transparence des comptes des sociétés et les réformes du code du commerce.

\subsection{Les mesures facilitant les investissements étrangers}

La création de l'OTO (Office of Trade and Investment Ombudsman ${ }^{11}$ ), en 1982, est la première mesure effective d'ouverture aux investissements étrangers. L'OTO a pour objectif de faciliter l'entrée des firmes étrangères sur le marché japonais. Sa mission consiste, d'une part, à recevoir les plaintes des firmes étrangères victimes de procédures discriminatoires (liées aux importations ou aux IDE entrants) et, d'autre part, à organiser des concertations

\footnotetext{
${ }^{10}$ Traditionnellement les F\&A sont difficiles au Japon même lorsque quelles sont réalisées entre firmes japonaises [JIC 1996].

${ }_{11}$ Il est à noter que le sigle de fait pas référence à l'investissement, ce qui montre qu'à sa création, le volet des investissements n'étant pas considéré comme important.
} 
interministérielles et coordonnées pour gérer ces plaintes ${ }^{12}$. Ses résultats sont limités car l’OTO s’intéresse exclusivement aux barrières à la frontière (qui ne sont pas toujours les plus pénalisantes pour les entreprises) et il semble avoir peu de pouvoir vis à vis de ministères importants.

Cependant, l'OTO est une exception car les premières mesures réellement significatives n'ont été prises qu’en 1992. La première est la révision de la "Foreign exchange law » afin de simplifier les IDE entrants. La seconde est la loi «Law on extraordinary measures for the promotion of imports and facilitation of foreign direct investment in Japan » (qui portait sur la période 1992-1995 et a été étendue à la période 1996-2006) ${ }^{13}$. Cette loi est une des plus importantes en matière d'ouverture aux investissements internationaux.

Cette loi revêt plusieurs aspects : elle prévoit une procédure de certification « d’investisseurs entrants » pour les firmes étrangères qui investissent au Japon. Celle-ci leur permet de bénéficier d'incitations fiscales et de garanties de prêts et d'obtenir des réductions de taux d'intérêt, elle comprend également la création, en 1993, du FIND (Foreign Investment in Japan Development Corporation) et des FAZ (Foreign Access Zones). Le FIND fournit divers services comme des services de conseil d'accès au marché, des services d'aide au recrutement afin de faciliter les activités des firmes étrangères. Depuis 1996 sa mission porte essentiellement sur les fusions et acquisitions et il propose des mesures concrètes d'aide à ces opérations ${ }^{14}$.

Les FAZ ont pour objectif d'aider les implantations de firmes et de capitaux étrangers. Ils concentrent des installations liées aux importations des entreprises et des activités, établies près des aéroports et des ports ${ }^{15}$. De plus des sociétés de services quasi publiques ont été établies pour augmenter les infrastructures de façon à promouvoir les investissements entrants

\footnotetext{
${ }^{12}$ Cet organisme a été saisi 584 fois depuis sa création jusqu'en 1998, dans 189 cas, des procédures ont été modifiées, dans 212 cas, les plaintes ont été abandonnées s'agissant de problèmes d'interprétation et dans les cas restant, les procédures en cause n'ont pas été modifiées. Les cas soumis à l'OTO sont en régression mais cela peut refléter une déception de la part des firmes étrangères qui semblent parfois craindre des mesures de représailles.

${ }^{13}$ Préalablement à cette loi certaines mesures ont été prises à la fin des années 80, notamment en 1986, "The private participation law" offrant des installations d'affaires temporaires pour les firmes. Les firmes opérant au Japon peuvent obtenir des prêts auprès des institutions financières japonaises (the japan development bank and Hokkaido-Tohoku development public corp).

${ }^{14}$ En novembre 1997, le FIND a étudié des mesures concrètes pour améliorer le climat d'accueil des fusions incluant de meilleures façons d'apporter de l'information et d'améliorer les procédures administratives obligatoires. Le JIC étudie également des mesures pour promouvoir les IDE entrants.

${ }^{15}$ Il existe actuellement 22 FAZ au Japon.
} 
(des installations pour aider la distribution d'importations, pour fournir de l'information etc). Ces activités sont qualifiées de «Import Infrastructure Improvment Activities ».

La loi prévoit enfin l'ouverture de centres de «support» aux affaires dans certaines agglomérations pour assister les hommes d’affaires durant la période d’entrée.

En plus de cette loi, différents programmes d'assistance et de supports sont fournis aux firmes étrangères. En 1994, le JIC (Japan Investment Council) est crée. Il a pour mission de promouvoir les investissements, de coordonner les politiques des ministères et des agences et de diffuser de l'information. En 1996, le JIC annonce que les fusions et acquisitions sont autorisées et que des mesures seront rapidement prises pour les favoriser (diverses mesures comme les réductions de taux d'intérêt, des simplifications administratives lors de fusions sont adoptées). Dans son rapport d'avril 1999 intitulé «sept recommandations pour promouvoir les IDE au Japon» le JIC recommande de poursuivre le processus de dérégulation.

Ainsi, le gouvernement japonais a cherché à ouvrir son marché aux firmes étrangères et à promouvoir les IDE entrants. Trois types d’action ont été menés :

- Une action visant à améliorer l'information des firmes étrangères (en tentant de rendre le marché plus transparent) en simplifiant et en réduisant les procédures administratives.

- Une action portant sur l'amélioration des conditions financières d'accès au marché (grâce à des réductions de taux d'intérêt ou à des garanties de prêt).

- Une action visant à fournir les infrastructures indispensables aux firmes pour accéder au marché japonais.

\section{Dynamique des investissements directs et des fusions et acquisitions au} Japon

L'étude du processus de déréglementation et des principales mesures prises par les autorités japonaises a permis de constater la réalité de l'ouverture de ce marché aux firmes étrangères. L'examen de l'évolution des flux d'IDE entrants au Japon fournit une évaluation de l'efficacité des mesures prises. Nous montrerons, dans un premier temps, que cette croissance s'est accélérée dans les dernières années et dans un second temps qu’elle s’est accompagnée d'une 
modification de la forme de ces investissements particulièrement pour ce qui concerne les F\&A.

\subsection{Les investissements directs étrangers au Japon : dynamique et diversification}

Les investissements directs au Japon, bien que relativement peu important quantitativement, ont historiquement joué un rôle central dans la structuration de l'industrie de ce pays. Ils ont notamment été à l'origine de la modernisation de l'industrie entre les deux guerres et le gouvernement a, pendant cette période, encouragé les entrées de firmes occidentales dans des secteurs aussi divers que la chimie, les pneumatiques et l'électromécanique [Takeshi \& Udagawa 1990, Bloström et alii 2000]. Après la seconde guerre et jusqu'au années 70, les investissements directs étaient très peu nombreux en raison de barrières officielles et officieuses à l'entrée des capitaux étrangers. Les barrières officielles ont été progressivement levées depuis la fin des années 80 mais l’accès au marché japonais reste encore difficile en raison des spécificités de cette économie. Depuis, 1992, des réformes ont été entreprises pour ouvrir l'économie japonaise aux capitaux étrangers.

La croissance des investissements directs de firmes étrangères est particulièrement forte depuis le milieu des années 90 (graphique 1). A partir de 1997, la valeur de ces opérations connaît une accélération importante, elles passent de 13,4 milliards de yens en 1998 (année fiscale 1998-1999) à près de 24 milliards de yens pour l'année fiscale 1999-2000. L’analyse comparée du nombre et de la valeur des opérations montre que la taille moyenne des opérations tend à augmenter sur l'ensemble de la période et plus particulièrement dans les dernières années. Cette évolution traduit vraisemblablement une modification des comportements d'entrée des firmes étrangères. Les opérations sont plutôt moins nombreuses mais leur taille augmente et elles prennent de plus en plus souvent la forme de F\&A. Pour les six premiers mois de l'année 2000, le nombre d'opérations d'IDE est de 853 pour un montant de 180 milliards de yens, soit une croissance de 43\% par rapport au six premiers mois de 1999 [Ministère japonais des finances 2001]. 
Graphique $\mathbf{n}^{\circ} 1$ : Evolution en nombre et en valeur (centaine de millions de yens) des IDE au Japon

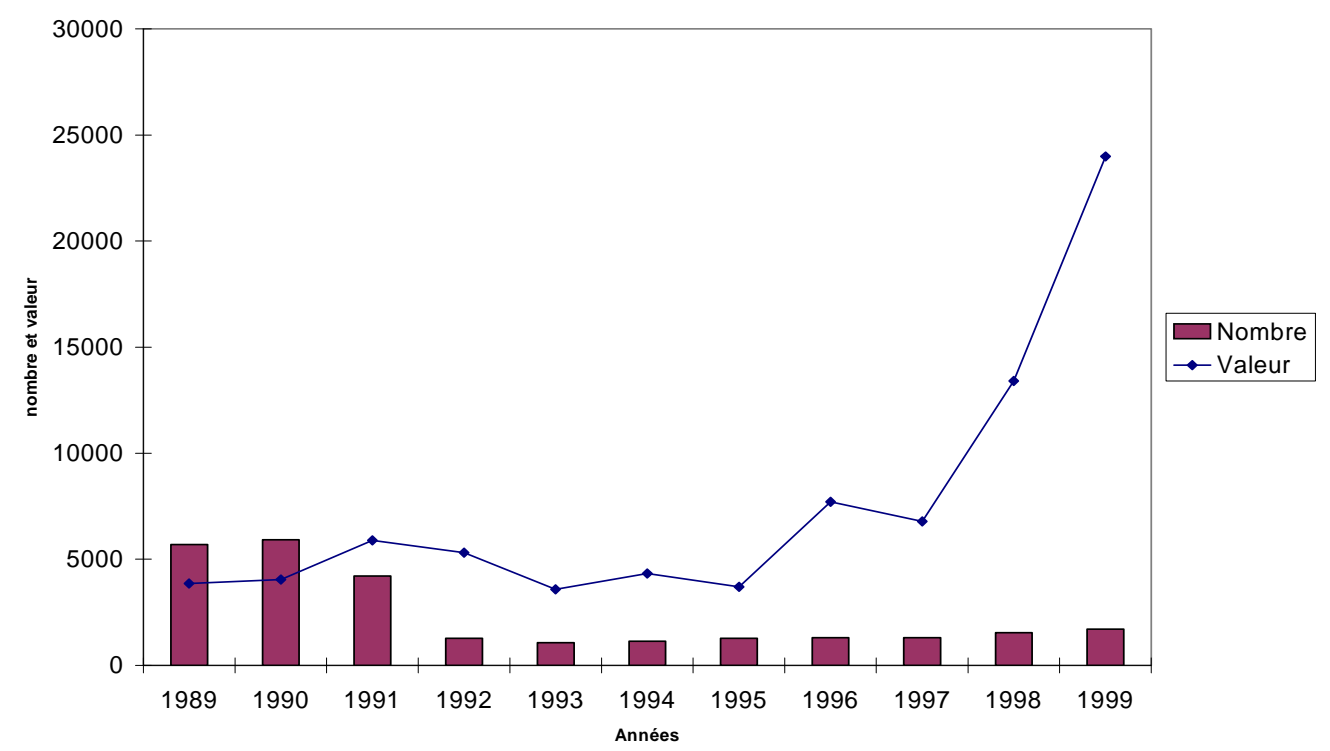

Sources : Ministère des finances du Japon [2001]

Une analyse des flux d'IDE entrants par grandes zones géographiques montre que depuis 1992, date de la première mesure significative d'ouverture, les firmes de l'ensemble des zones développées ont sensiblement augmenté leurs investissements au Japon. Cependant, le tableau 2 indique que les firmes originaires du continent nord américain et de l'Europe sont les plus offensives, devançant ainsi les autres pays asiatiques. En 1999, les firmes européennes ont très fortement augmenté leurs opérations et dépassent largement les firmes américaines. Parallèlement les firmes originaires des pays de l'Asie du Sud Est ont, en raison de la crise asiatique, ralenti leurs opérations. La comparaison des résultats des six premiers mois de 2000 par rapport à ceux des six premiers mois de 1999, met en évidence le poids des opérations exceptionnelles. L’Amérique du Nord a triplé la valeur de ses opérations, l’Amérique du Sud l'a multiplié par six et l'Europe connaît une baisse de 91\% [Ministère japonais des finances 2001]. 
Tableau $\mathbf{n}^{\circ} 2$ : Valeur des investissements directs entrant au Japon (en milliards de yens)

\begin{tabular}{|c|c|c|c|c|c|c|c|c|c|}
\hline & 1992 & 1993 & 1994 & 1995 & 1996 & 1997 & 1998 & 1999 & $\begin{array}{l}2000 \\
(6 \\
\text { mois })\end{array}$ \\
\hline Amérique du Nord & 183,7 & 126,2 & 198,1 & 178,6 & 244,6 & 152,1 & 809,5 & 417,3 & 660,1 \\
\hline Europe & 190,1 & 119,9 & 158,6 & 127,4 & 220,2 & 307,8 & 302,3 & 1413,7 & 92,4 \\
\hline Asie & 12,8 & 53,9 & 27,1 & 24,7 & 137,2 & 74,2 & 21,1 & 110 & 15,8 \\
\hline Amérique latine $^{16}$ & 98,1 & 17,3 & 13,6 & 14,1 & 65,6 & 59,1 & 34,3 & 289,5 & 131,2 \\
\hline $\begin{array}{l}\text { Proche et Moyen } \\
\text { Orient }\end{array}$ & 1,3 & 0,3 & 0 & 0 & 2 & 0,1 & 0,1 & 0,2 & 0,2 \\
\hline Afrique & 1,6 & 0,5 & - & 1 & - & 0 & 0 & 0,4 & 0,2 \\
\hline Océanie & 0 & 1,2 & 3,4 & 0,5 & 1,4 & 0,7 & 0,1 & 6,8 & 6,4 \\
\hline
\end{tabular}

Sources : Ministère des finances du Japon 2001

L'examen des opérations par pays confirme et précise les résultats précédents (tableau 3). Les pays dont les investissements sont les plus importants sont les États-Unis, la France, les PaysBas et le Canada. Cependant, le poids des grandes opérations pèse fortement sur la hiérarchie des pays. Ainsi, les États-Unis qui étaient de loin le premier pays investisseur au Japon en 1998 ont été dépassé par la France en 1999. La valeur élevée (33 milliards de francs) de l'opération Renault-Nissan explique vraisemblablement ce résultat. Au sein le l’Europe, les Pays-Bas sont un investisseur majeur au Japon ${ }^{17}$, suivi par le Royaume-Uni et l'Allemagne. Les résultats pour les six premiers mois de 2000 bouleversent cette hiérarchie avec un triplement des investissements des Etats-Unis, une baisse très importante pour le Canada (97,5\%), la France (-97,3\%) et l'Allemagne (-97,4\%) [Ministère japonais des finances 2001].

\footnotetext{
16 Les investissements originaires d’Amérique latine proviennent, pour la quasi totalité, des îles Caïman et des îles vierges.

${ }_{17}$ Cette forte présence des Pays-Bas s'explique essentiellement par l'utilisation par des firmes d'autres pays, et pour des raisons fiscales, de cette nationalité.
} 
Tableau 3 : Les investissements directs au Japon des principaux pays développés entre 1998 et 2000

\begin{tabular}{|c|c|c|c|c|c|c|}
\hline \multirow{2}{*}{} & \multicolumn{2}{|c|}{1998} & \multicolumn{2}{c|}{1999} & \multicolumn{2}{c|}{2000 (6 mois) } \\
\cline { 2 - 7 } & Nombre & $\begin{array}{c}\text { Valeur en } \\
\text { milliards } \\
\text { de yens }\end{array}$ & Nombre & $\begin{array}{c}\text { Valeur en } \\
\text { milliards de } \\
\text { yens }\end{array}$ & Nombre & $\begin{array}{c}\text { Valeur en } \\
\text { milliards de } \\
\text { yens }\end{array}$ \\
\hline Etats-Unis & 624 & 807,8 & 622 & 248,7 & 296 & 659,9 \\
\hline Canada & 11 & 1,7 & 12 & 168,6 & 6 & 0,2 \\
\hline Allemagne & 106 & 33,5 & 82 & 46,7 & 33 & 10,5 \\
\hline France & 36 & 16,8 & 49 & 745,7 & 29 & 15,6 \\
\hline Italie & 9 & 4,4 & 16 & 1,2 & 2 & - \\
\hline Royaume- & 6 & 37 & 65 & 89,8 & 45 & 6,5 \\
Uni & & & & & & 17,8 \\
\hline Pays-Bas & 86 & 128 & 104 & 471,2 & 44 & \\
\hline
\end{tabular}

Sources : Ministère des finances du Japon [2001]

En terme de stocks d'IDE, c'est à dire en cumulant les opérations réalisées depuis 1950, l'Europe et l'Amérique du Nord font jeu égal et représentent près de $80 \%$ des investissements directs au Japon. Les Etats-Unis sont particulièrement représentés suivis des Pays-Bas et de la France.

Tableau 4 : Les stocks d'investissements directs au Japon en 1999

\begin{tabular}{|l|c|c|}
\hline & Stocks en milliards de yens & Part en \% \\
\hline Etats-Unis & 2901,9 & 36,7 \\
\hline Pays Bas & 1000,4 & 12,7 \\
\hline France & 712,9 & 9 \\
\hline Allemagne & 374,2 & 4,7 \\
\hline Royaume-Uni & 359,4 & 4,6 \\
\hline Suisse & 315,5 & 4 \\
\hline Canada & 181,2 & 2,3 \\
\hline Hong Kong & 102,3 & 1,3 \\
\hline
\end{tabular}

Sources : d'après Ministère des finances du Japon et PEE et l'Ambassade de France au Japon 
L'analyse sectorielle (graphiques 2 et 3) montre que les IDE entrants sont inégalement répartis au sein de l'économie japonaise.

Dans le secteur manufacturier ils sont, notamment à partir de 1997, concentrés dans les secteurs chimique et mécanique ${ }^{18}$. Pour le premier semestre 2000, seule l'industrie chimique connaît une croissance significative des opérations d’IDE (+ 277\%)[Ministère des finances du Japon 2001].

\section{Graphique 2 : Évolution des IDE dans les secteurs manufacturiers}

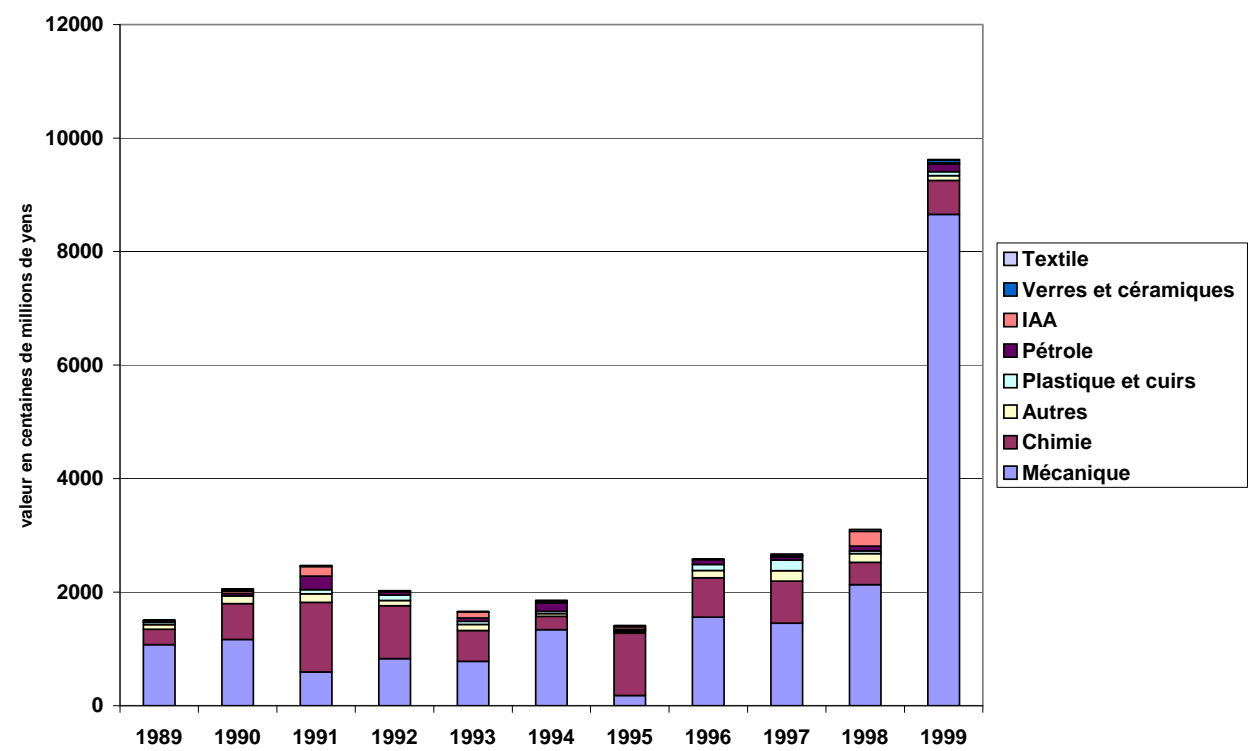

Sources : Ministère des finances du Japon [2001]

La forte croissance, à la fin de la décennie 90, des opérations dans le secteur non manufacturier, s'accompagne d'une diversification des secteurs d'activités dans lesquels se diffusent les IDE. Les secteurs de la banque-finance, des télécommunications, du commerce et de la construction sont désormais largement concernés. Ces secteurs sont également ceux pour lesquels les réformes réglementaires ont été les plus importantes. Le premier semestre de 2000 confirme cette tendance avec une croissance importante de la valeur des opérations réalisées dans le secteur de la banque et de l'assurance (+ 769\%), l'immobilier (+ 403\%) et les télécommunications (+ 132\%). 


\section{Graphique 3 : Évolution des IDE dans les secteurs non manufacturiers}

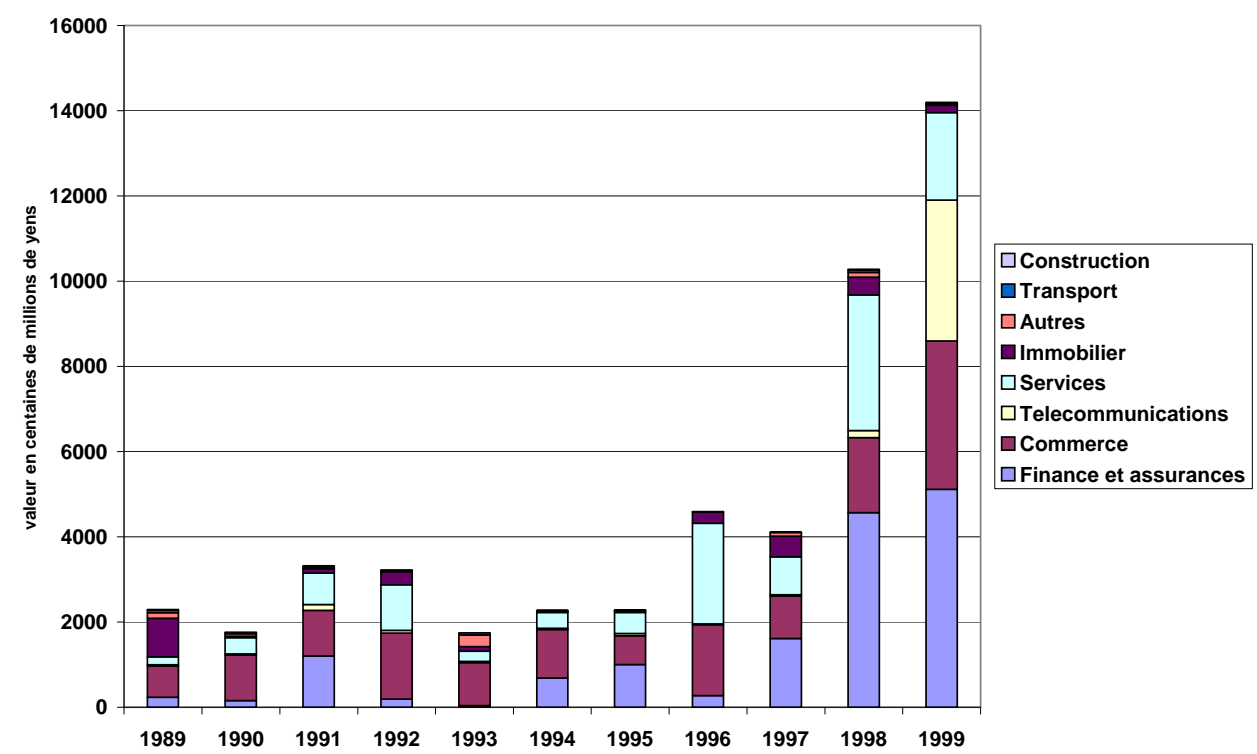

Sources : Ministère des finances du Japon [2000]

\subsection{Les fusions et acquisitions au Japon}

Au sein des IDE, nous avons porté une attention particulière aux opérations de F\&A. En effet, cette forme d'investissement rendue possible grâce aux modifications réglementaires traduit un changement du comportement des firmes étrangères. Le nombre d'opérations de F\&A a connu une forte augmentation pendant la seconde partie de la décennie 80 suivie d'une décroissance pendant la première partie des années 90 puis à nouveau une forte augmentation depuis 1993 (graphique 4). Au sein de cette tendance générale, les évolutions des différentes formes de F\&A sont relativement contrastées. Ainsi, le nombre d'opérations engagées par les firmes japonaises vers l'étranger (F\&A sortantes) suit assez fidèlement jusqu'en 1994 la tendance générale constatée ci-dessus puis stagne. Cette situation s'explique en grande partie par la crise japonaise et résulte des difficultés de plus en plus importantes rencontrées par les firmes japonaises pour financer leur expansion à l'étranger et par l'effort engagé dans la restructuration de leurs activités au Japon. Cet effort de restructuration est mis en évidence par les opérations de F\&A internes (entre firmes japonaises). Jusqu'en 1995, elles connaissent une évolution tendanciellement moins marquée que le total des opérations puis elles augmentent de manière importante et représentent, à la fin de la période, plus de $60 \%$ du total des F\&A. Cette dynamique est due aux évolutions législatives et à la recomposition de l'appareil

\footnotetext{
${ }^{18}$ En 1999 l'opération entre Renault et Nissan accroît artificiellement la concentration dans le secteur de la mécanique.
} 
productif japonais consécutif à la crise. La crise a incité les firmes japonaises à se recentrer sur leur activité de base et à vendre les activités considérées comme moins stratégiques, ceci contribuant à augmenter les opportunités de F\&A. Les opérations de F\&A des firmes étrangères (entrantes) ont connu une croissance très faible pendant la période 1985-1995 puis une forte accélération (qu'il faut toutefois relativiser compte tenu du faible niveau de départ) après cette date. Elles représentent en 1999 environ 11\% du total des opérations de F\&A contre 2,5\% en 1990 .

\section{Graphique 4 : Évolution du nombre d'opérations de F\&A au Japon}

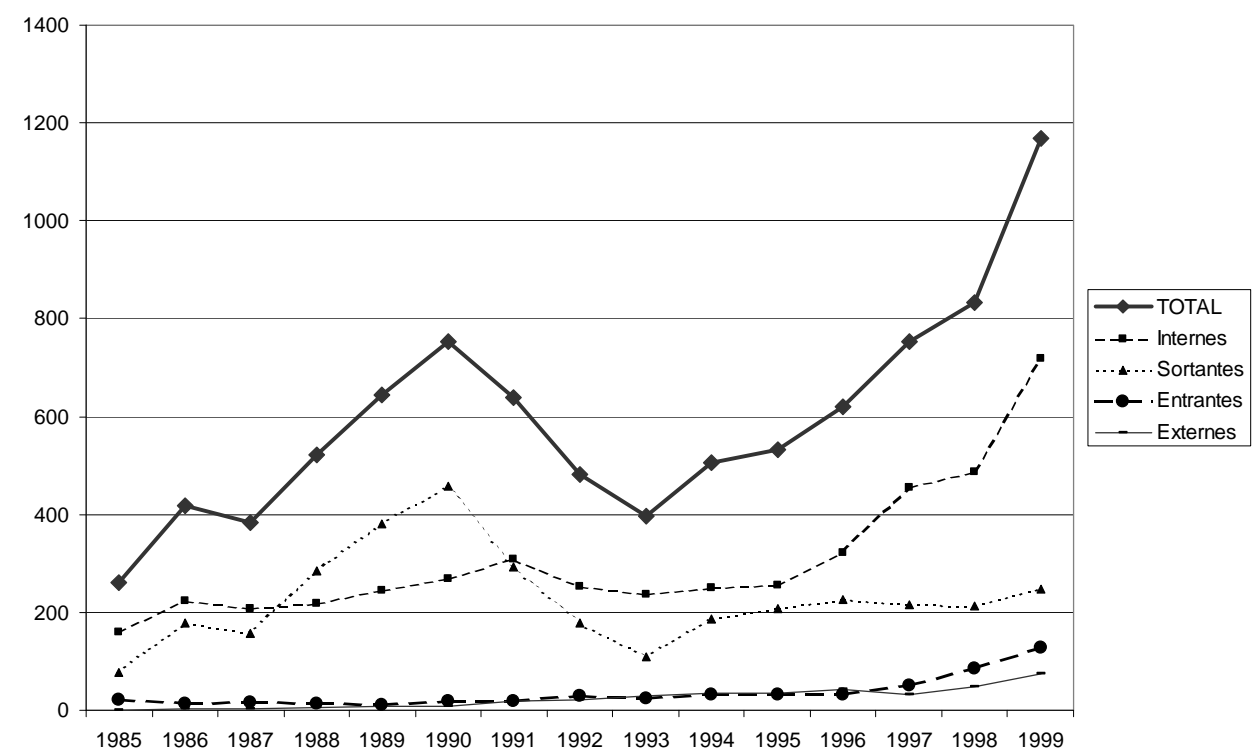

Sources : d'après JETRO [2000]

L'évolution des performances de l'économie japonaise a incontestablement modifié les dynamiques des différentes formes de F\&A. A la fin des années 80, ces opérations accompagnaient l'expansion à l'étranger des firmes japonaises qui cherchaient à réduire leurs coûts de production (aggravés par la forte appréciation du yen) mais aussi à contourner des mesures protectionnistes par des investissement directs à l'étranger (industries automobile et électronique notamment). Ce mouvement s'est ralenti et a été relayé par des opérations internes de restructuration de l'appareil productif japonais. A la fin des années 90, la croissance significative des opérations de F\&A entrantes s'explique par la plus forte attractivité du marché japonais due aux difficultés des firmes japonaises mais aussi aux premiers résultats de la politique d'ouverture que nous avons vu précédemment. Cependant les opérations de F\&A internationales sont très nettement moins importantes au Japon que dans 
les autres pays développés (tableau n5). En 1999, le montant des achats japonais par F\&A a considérablement augmenté par l'intermédiaire d'une seule opération. De façon générale, les firmes japonaises ont peu recours à ces opérations pour leur développement à l'étranger. En revanche, ces dernières années, les ventes effectuées par l'intermédiaire de F\&A se sont développées rapidement et ont dépassé les achats au cours de la période 1997-1999.

Tableau 5 : Fusions et acquisitions internationales :

ventes et achats dans les pays développés, 1990-1999

(en Milliards de dollars)

\begin{tabular}{|l|l|l|l|l|l|l|l|l|l|l|l|}
\hline Pays/régions & \multicolumn{5}{|c|}{ Ventes } & \multicolumn{5}{c|}{ Achats } \\
\hline & $\mathbf{1 9 9 0}$ & $\mathbf{1 9 9 5}$ & $\mathbf{1 9 9 7}$ & $\mathbf{1 9 9 8}$ & $\mathbf{1 9 9 9}$ & $\mathbf{1 9 9 0}$ & $\mathbf{1 9 9 5}$ & $\mathbf{1 9 9 7}$ & $\mathbf{1 9 9 8}$ & $\mathbf{1 9 9 9}$ \\
\hline $\begin{array}{l}\text { Pays } \\
\text { Développés } \\
\text { dont }\end{array}$ & $\mathbf{1 3 4 , 2}$ & $\mathbf{1 6 4 , 6}$ & $\mathbf{2 3 4 , 7}$ & $\mathbf{4 4 5 , 1}$ & $\mathbf{6 4 4 , 6}$ & $\mathbf{1 4 3 , 2}$ & $\mathbf{1 7 3 , 7}$ & $\mathbf{2 7 2}$ & $\mathbf{5 5 1 , 4}$ & $\mathbf{6 7 7 , 3}$ \\
\hline $\begin{array}{l}\text { Union } \\
\text { européenne }\end{array}$ & 62,1 & 75,1 & 114,6 & 187,9 & 344,5 & 86,5 & 81,4 & 142,1 & 284,4 & 497,7 \\
\hline Etats- Unis & 54,7 & 53,2 & 81,7 & 209,5 & 233 & 27,6 & 57,3 & 80,9 & 137,4 & 112,4 \\
\hline Japon & 0,1 & 0,5 & 3,1 & 4 & 15,9 & 14 & 3,9 & 2,7 & 1,3 & 9,8 \\
\hline
\end{tabular}

Sources : Rapport sur l'investissement dans le monde [CNUCED 2000]

Les résultats de l'analyse sectorielle (graphique 5) sont conformes à ceux présentés pour les IDE. Cependant, il existe une très forte disparité entre les opérations de type interne et entrant. Les opérations internes se concentrent, en 1999, dans la distribution, les services et la banque. L'importance des opérations réalisées ces secteurs montre que les efforts de déréglementation semblent conduire à des mouvements de restructuration. En ce qui concerne les opérations entrantes, le secteur financier (banques et "autres institutions financières") ainsi que la mécanique et l'électromécanique sont les activités les plus engagées. La crise du système financier japonais et les importantes réformes de déréglementation expliquent en grande partie le dynamisme dans le premier secteur. 


\section{Graphique 5 : Répartition sectorielle des opérations de F\&A en 1999}

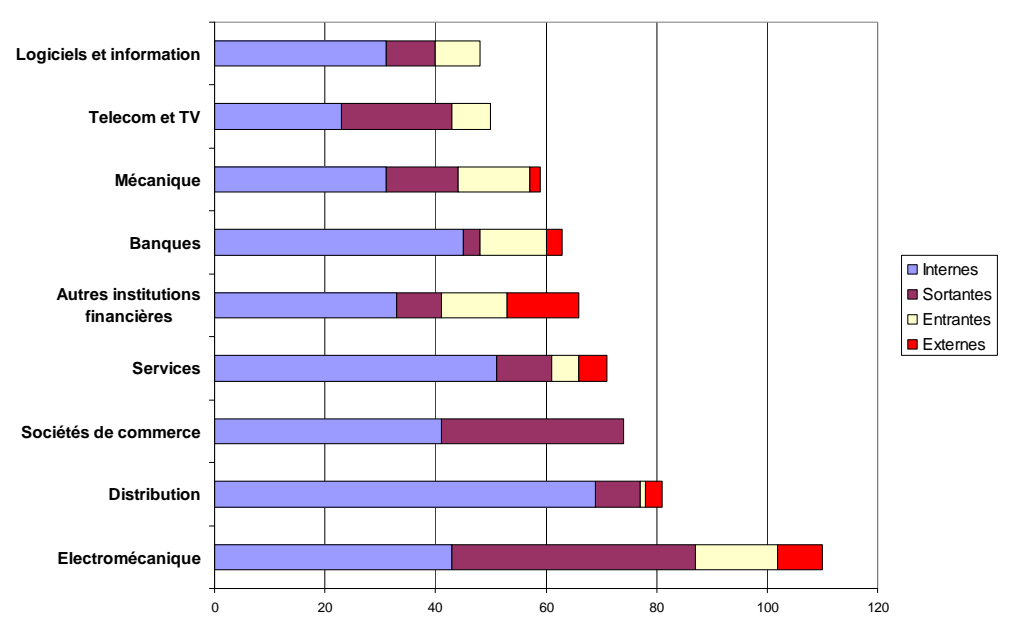

Sources: d'après JETRO [2000]

Une distinction entre les différentes formes d'opérations ${ }^{19}$ permet de constater que les opérations d'acquisition (majoritaires et minoritaires) et les acquisitions d'une activité ont connu une croissance importante dans les dernières années (graphique 6). Cette tendance confirme l'importance des opérations de restructuration. En effet, celles-ci obligent les firmes acheteuses à sélectionner de manière précise les actifs dont elles ont besoin.

${ }^{19}$ Les fusions, les acquisitions majoritaires dans lesquelles la firmes acheteuses acquiert plus de $50 \%$ du capital de sa cible, les acquisitions minoritaires majoritaires dans lesquelles la firmes acheteuses acquiert mois de 50\% du capital de sa cible, les acquisitions d'une activité où la firmes achète une partie des actifs de la société - cible, les augmentations de participation où la firme augmente sa participation dans le capital de l'entreprise - cible sans atteindre 50\%. 


\section{Graphique 6 : Les opérations de F\&A par types}

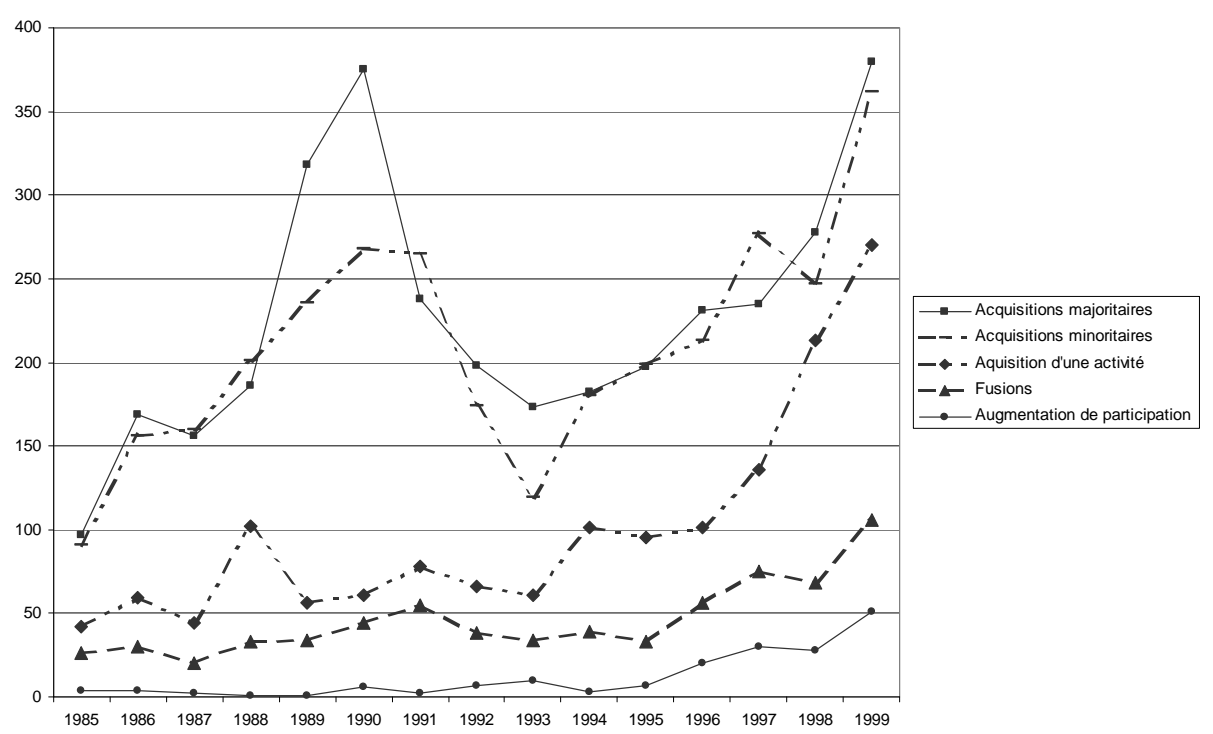

Sources : d'après JETRO [2000]

La seconde moitié des années 90 est marquée par une forte croissance des investissements étrangers au Japon. Cette tendance s'est accentuée en 1999 qui apparaît comme une année record en ce qui concerne la pénétration des capitaux étrangers. Ces investissements proviennent principalement des pays développés et en particulier de l'Amérique du Nord et du Japon. La crise économique et financière survenue en Asie a freiné les investissements de cette zone. L'Europe et surtout la France ont commencé à rattraper leur retard et ont multiplié les opérations dans ce pays. Une attention particulière portée aux F\&A a permis de montrer non seulement que cette forme d'investissement se développait mais que les stratégies des firmes qui pénétraient ce marché étaient en train de changer. En effet, la croissance des F\&A suit assez fidèlement celle des IDE car les firmes ont recouru massivement à cette forme d'opérations. 


\section{$\underline{\text { Conclusion }}$}

Les réformes entreprises au Japon et la crise économique que traverse ce pays ont incontestablement conduit à l'ouverture d'un marché traditionnellement fermé. Nous avons, dans un premier temps, dressé un inventaire des principales mesures adoptées en montrant qu'elles couvrent un champ très large. Ce processus de déréglementation a été long et particulièrement complexe. Il aura, par exemple, fallu plus de 10 ans pour que la modification de la loi sur les grandes surfaces soit effective. En outre, toutes les réformes engagées ont fait l'objet de nombreuses discussions et rapports pour aboutir à une multitude de mesures très partielles portant sur des domaines d'application précis. Le programme de 1995 comporte ainsi plus de 2800 réformes de portée souvent restreinte.

Nous avons, dans un second temps, montré que ces évolutions réglementaires se sont accompagnées d'une forte augmentation des IDE. Ces investissements prennent souvent la forme d'opérations de fusions et d'acquisitions qui concernent un spectre de plus en plus large d'activités. La croissance de ces investissements étrangers, quel que soit leur forme, a été souvent plus forte dans les secteurs ayant connu des réformes spécifiques (comme la banque, l'assurance ou les services).

Cette étude confirme l'ouverture des firmes japonaises aux capitaux étrangers et montre qu'elle a été rendue possible grâce au processus de déréglementation. Ainsi, les firmes étrangères ont participé aux opérations de restructuration de l'appareil de production japonais de la fin des années 90. Cette participation reste modeste mais n'est plus insignifiante. 


\section{Bibliographie}

Ambassade de France au Japon [2000] service d'expansion économique, les entreprises françaises au Japon

BLOMSTRÖM M. [1989] Foreign investments and slipover, London, Routledge

CARLILE L.E. \& TILTON M.C. eds [1988] Is Japan really changing its ways? Regulatory reform and the Japanese economy, Washington DC. Brookings.

DUNNING J [1996] "Explaining Foreign investment in Japan : some theoretical insights" in M; YOSHITOMI and E. GRAHAM (eds), Foreign direct investment in Japan, Cheltenham, Edward Elgar.

ENCARNACION D [199]2 Rivals beyond trade : America versus Japan in global competition, Ithaca : Corbvell University Press.

Fusion et Acquisition décembre 1989-Janvier 2000

JETRO (Japan External Trade Organisation), [1999-1], Direct investment in Japan, Investment news, $\mathrm{n}^{\circ} 18$

JETRO, [1999-2] White paper on foreign, direct investment

JETRO [2000-1] le Japon à la page, n²9, juin

JETRO [2000-2] “Business opportunities in Japan”, Investment news, n²2

JIC (Japan Investment Council) [1996] On the preparation of M\&A environment in Japan

JIC [1999-1] Seven recommendations for promoting FDI in Japan

JIC [1999-2] Yearbook

LAWRENCE R.Z. [1987] “import in Japan : closed markets or minds “?, Brooking papers on economic activity, $1987 \mathrm{n}^{\circ} 2$

Ministère des Finances du Japon (MOF) www.mof.gov.jp

MITI [1998] White paper on trade and industry

OCDE [1999] La réforme de la réglementation au Japon

SAXONHOUSE G.R. [1993] "What does Japanese trade structure tells us about Japanese policy”, Journal of Economic Perspectives, vol 7 n³, pp 21-23. 
SOHN Y. (1998) "The rise and development of the Japanese licensing system" in CARLILE L.E. \& M.C. TILTON, eds. Is Japan really changing its ways? Regulatory reform and the Japanese economy, Washington DC. Brookings.

TAKESHI Y. and M. UDAGAWA eds. [1990] Foreign business in Japan before world war II, Tokyo, University of Tokyo Press.

WEISTEIN D. [1997] "Foreign direct investment and kereitsu: rethinking US and Japanese policy” in FEENSTRA R. eds, Effects of US trade and promoting policies, Chicago, Chicago University Press.

YOSHITOMI M and GRAHAM E. eds [1996], Foreign direct investment in Japan, Cheltenham, Edward Elgar. 